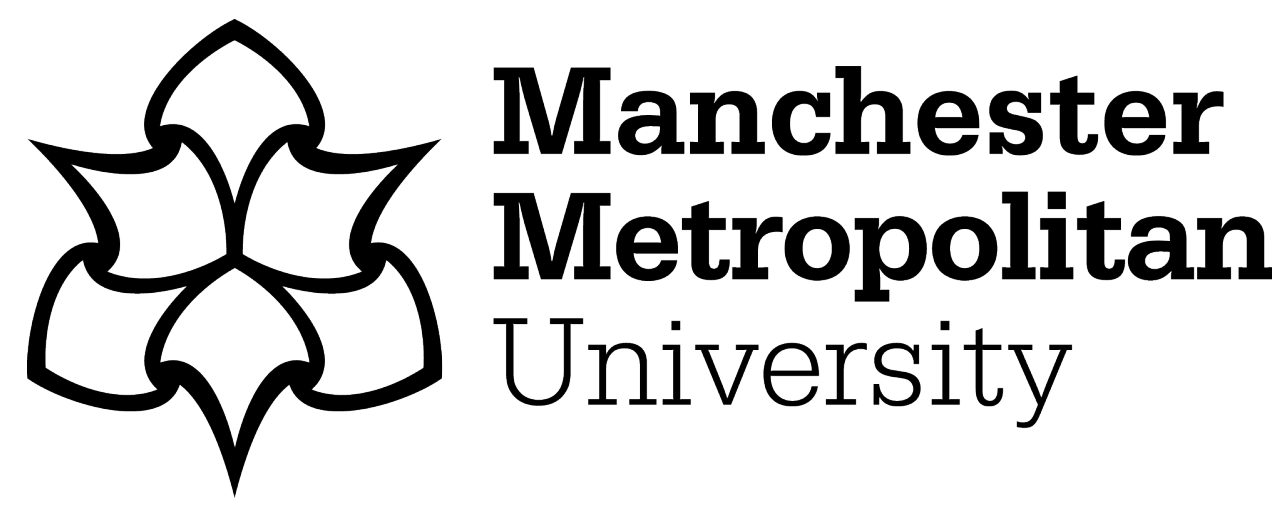

Gheth, W, Rozman, M, Rabie, KM and Adebisi, B (2018) EMC Measurements in Indoor Power Line Communication Environments. In: International Telecommunications Conference, 28 September 2018 - 29 September 2018, Istanbul, Turkey.

Downloaded from: https://e-space.mmu.ac.uk/621263/

Version: Accepted Version

Publisher: Springer, Singapore

DOI: https://doi.org/10.1007/978-981-13-0408-8_16

Please cite the published version 


\title{
EMC Measurements in Indoor Power Line Communication Environments
}

\author{
Waled Gheth, Matjaz Rozman, Khaled M. Rabie, Bamidele Adebisi \\ School of Engineering, Manchester Metropolitan University, UK, M1 5GD, \\ waled-ali.m.gheth@stu.mmu.ac.uk.
}

\begin{abstract}
The transmit power of communication signals over power lines is limited due to restrictions imposed by regulatory bodies and standards. In this paper, the electromagnetic emissions from two different power line communication (PLC) modems are measured in different indoor environments in the frequency range between $1 \mathrm{MHz}$ to $30 \mathrm{MHz}$. The obtained measurements are compared with the current available limits and regulations. Also, a comparison between the emission measurements of the two modems is made. Results have shown that, in some scenarios, the measured emissions do not comply with some of the available standards. Based on this, recommendations are made towards the end of the paper.
\end{abstract}

Keywords: Electromagnetic comparability (EMC), measurements, power line communication (PLC), regulations and standards.

\section{Introduction}

Power lines have always been a means to distribute electrical power. However, with the rising dependence on communications, power line networks were seen as a possible medium for delivering data. This technology is commonly known as power line communication (PLC). PLCs exploit the existing power cables in buildings or utility grid to simultaneously carry communication signals and deliver electricity. PLCs can play an important role in home networking applications due to the spreading of internet and modern communication technologies. However, the power line network was initially designed to transmit alternating current (AC) to consumers at frequencies $50 \mathrm{~Hz}$ and $60 \mathrm{~Hz}$; hence, several challenges are faced when using this medium for high-data rate transmissions $[2,7,9]$. Among these is the electromagnetic interference (EMI) issue due to the fact that at high frequencies power lines tend to act as radiating antennas [1]. There are two types of the EMI, namely, inductive and conductive. A number of standards and limits for the two types have been set by different standardization bodies, for more details the reader may refer to [1]. [3]

Power lines radiate EMI to the surrounding as communication signals travel along the wires and electronic equipment malfunction might occur [11]. It is known that the non-shielded electrical cables act as an effective transmission 
antenna at higher frequencies ( $>1 \mathrm{MHz}$ spectrum) utilized in PLC networks [8]. However, the interference caused by such signals is low in power, yet local consumers still have their own reason to complain. This local interference can be criticized by the amateur radio, who live or work near PLC systems [8]. They believe that sending data via power lines will interfere with the sensitive wireless systems being used.

As signals travel over power lines, they cause unacceptable risks in many areas of technology due to their radiation emissions. This interference should be controlled and kept at acceptable levels. Many studies on the topic of electromagnetic compatibility (EMC) have been reported in the literature, see e.g., [1,6,11]. In simple terms, EMC describes the capability of system devices to function in the surrounding electromagnetic environment with no error. Ensuring the reliability and safety of systems when they are used in electromagnetic environments is the main aim of EMC regulations. Practically, the electromagnetic disturbances caused by every single appliance on the network should be limited, also an appropriate level of immunity for each device to these disturbances must be considered [5].

Motivated by the above, in this paper, we will review and compare the common EMC regulations and standards. In addition, some measurements will be conducted in different indoor environments to measure electromagnetic emissions from two different PLC modems, unlike $[1,3]$ which considered only one environemnt and one modem. Experimental measurements are compared with the limits and regulations. Results show that the PLC modems under study mostly comply with the EMC standards but in some scenarios the measured radiated emissions were found to exceed the limits at some frequencies.

The rest of the paper is organized as follows. Section 2 presents and reviews the previous work done on this topic. Section 3 compares and discusses the common EMC standards. In Sections 4 and 5, we discuss the measurement methods and analysis of the approaches adopted in our work. Results are presented in Section 6 and, finally, conclusions are drawn in Section 7.

\section{Previous Work}

A number of radiated and conducted emission measurements were carried out in previous years to find out whether the PLC links comply with EMC standards. Different frequency ranges and environments were investigated. Some of the outcomes of these studies were promising and showed that PLC systems comply with the standards while others showed that there is incompliance existed [1]. Other studies investigated the possibility of extending the frequency range of PLC systems such as [10].

In [1], the measurements were taken within the frequency range from $1 \mathrm{MHz}$ to $30 \mathrm{MHz}$ in an indoor power line environment. The radiated emission measurements were carried out at a distance of $3 \mathrm{~m}$ away from the power line using an active loop antenna and the results were then compared to the Federal Communications Commission (FCC) standard. The differential and common modes 
of the conducted emission readings were taken using a current probe connected to a spectrum analyzer. The busy state of the common mode was compared to the EN55022 (main port and common mode). However, the comparison between the test outcomes and the standards showed that the measured results do not totally comply with the specified limits.

The possibility of extending the frequency band limits above $30 \mathrm{MHz}$ considering the EMC standards was investigated in [10]. A biconical antenna was used to measure the electric field at a distance of $3 \mathrm{~m}$ away from a $15 \mathrm{~m}$ power line installed in an open area site. Different signals in the frequency bands $2 \mathrm{MHz}-30 \mathrm{MHz}$ and $30 \mathrm{MHz}-100 \mathrm{MHz}$ were injected to the PLC network and EMC measurements were conducted. Results showed that the power spectral density (PSD) should be at a certain level in the frequency range $30 \mathrm{MHz}-100 \mathrm{MHz}$ in order to meet the EMC limits [10].

\section{Overview of EMC Standards}

EMC certificate is a topic that has been and will always be discussed in PLC systems. A different set of EMC standards exist for PLC technologies according to their operating frequency bands. Technologies that operate at frequencies 500 $\mathrm{kHz}$ and below are known as narrow band PLC, whereas those technologies using frequencies above $1.6 \mathrm{MHz}$ are referred to as broadband PLC (BB-PLC) systems [4]. However, frequency bands from $1.6 \mathrm{MHz}$ to $30 \mathrm{MHZ}$ are recommended by European Telecommunications Standards Institute (ETSI) for PLC systems [1]. This framework concentrates mostly on the BB-PLC technology and its relevant standards.

The issue of the EMC standards is still discussed by several standardization bodies. The International Special Committee on Radio Interference (CISPR) is an organization that plays a fundamental role in the context of EMC. It is a nongovernmental organization and was established in 1934 to set standards for controlling EMI. The CISPR is a part of the International Electrotechnical Commission (IEC). However, the standards that have been set by CISPR should be amended by local authorities in order to have legal effects [4].

Furthermore, European Committee for Electrotechnical Standardization (CENELEC) is the organization that is responsible for European standardization in this area. This organization started from CISPR standards and produced the European Norms (EN), which is called harmonized standards. These standards are then amended by local committees in EU and also have been adopted by the US standardization body, i.e., FCC. FCC Part 15 and CISPR 22, which were published in 1979 and 1985 respectively, are the two EMC standards that have an impact on PLC. The former is valid for commercial products marketed in the US and the latter is for outside the US. Nevertheless, CENELEC adopted the specific standards CISPR 22 with the norm EN55022 for the EU [4]. 


\section{Methods of Measurement and EMC Limits}

The conduced and radiated emissions are two classes of the EMC. The interference that is conducted through the power line is called the conducted emission whereas those caused by electric and magnetic fields produced by appliances and that propagate outside the power conductors are referred to as the radiated emissions. According to EN55022, the radiated emissions are only measured above $30 \mathrm{MHz}$, while the conducted emissions ought to be measured between the frequencies $150 \mathrm{KHz}$ and $30 \mathrm{MHz}$. Recently, measurements below $150 \mathrm{KHz}$ have been specified by the norm EN50065. However, FCC in the US includes that radiated emissions should be measured below and above $30 \mathrm{MHz}$ [4].

Each of the standardization bodies has its own limits and measurement setups. For instance, FCC specifies radiated and conducted limits in $\mu \mathrm{V} / \mathrm{m}$ and $\mu \mathrm{V}$, respectively, and the measurement is made at distance of $30 \mathrm{~m}$ using quasi-peaks level. NB30 standard uses a peak detector at distance of $3 \mathrm{~m}$ and the radiated limits are specified in $\mathrm{dB \mu V} / \mathrm{m}$. Modem operating mode during the measurement is another crucial factor. While EN55022 specifies a normal operating mode for measurement setup, both FCC and NB30 specify maximum emissions mode for measurement setup.

The conducted emission is distinguished among the terminal port and the telecommunication port in the EN5022 standard, where the former indicates the power supply and the later refers to the data transmission port. However, two modes are defined and considered in the conducted emission, namely, differential mode and common mode. Differential mode is related to the flow of the generated current from the phase conductor $(\mathrm{P})$ to the neutral conductor $(\mathrm{N})$ whereas the common mode is associated with the current flow through the phase conductor $(\mathrm{P})$ and neutral conductor $(\mathrm{N})$ synchronously then returns through the Protective Earth (PE) conductor [4]. In Fig. 1 we present these modes as used in our experiments (more details will be provided later). 


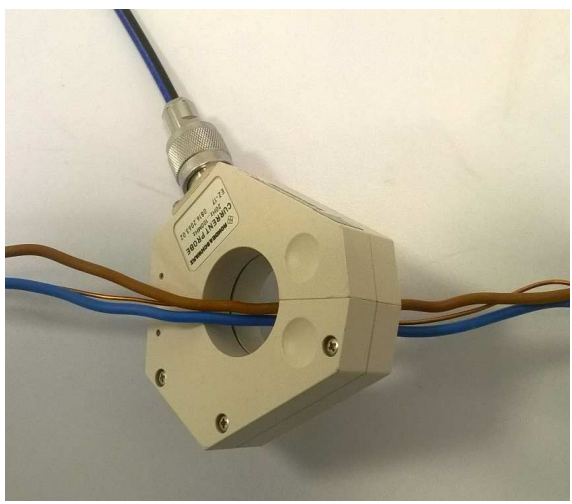

(a) Common mode measurement.

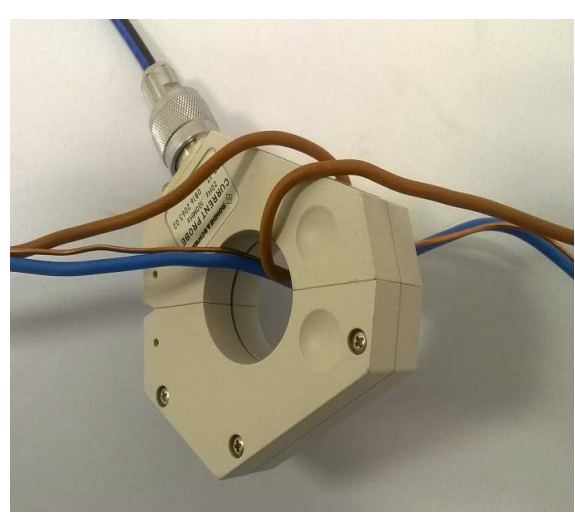

(b) Differential mode measurement.

Fig. 1: The real setups of the common and differential modes used in our measurements.

It can also be observed that in EN5022, devices are split into two classes: class A and class B. The devices that are used in industrial or commercial environments are specified as class A while the ones used in residential environments are called class B. However, EN5022 specifies class A limits with less strength than that of class B. For example, the class B quasi peaks (QP) main port limit for the frequency range $5 \mathrm{MHz}-30 \mathrm{MHz}$ is $60 \mathrm{~dB} \mu V$, while the same condition limit in class $\mathrm{A}$ is $73 \mathrm{~dB} \mu V$. For better understanding and clarity, we present in Table 1 (shown at the top of the next page) some differences in the limits of class A and class B devices [4].

\begin{tabular}{|c|l|c|c|c|c|c|c|c|}
\hline & \multicolumn{4}{|c|}{ Class A } & \multicolumn{4}{c|}{ Class B } \\
\hline \hline & main port limits & telecom limits & main port limits & \multicolumn{2}{c|}{ telecom limits } \\
\hline Frequencies & $\begin{array}{l}\mathrm{QP} \\
(\mathrm{dB} \mu V)\end{array}$ & $\begin{array}{c}\mathrm{AV} \\
(\mathrm{dB} \mu V)\end{array}$ & $\begin{array}{c}\mathrm{QP} \\
(\mathrm{dB} \mu V)\end{array}$ & $\begin{array}{c}\mathrm{AV} \\
(\mathrm{dB} \mu V)\end{array}$ & $\begin{array}{c}\mathrm{QP} \\
(\mathrm{dB} \mu V)\end{array}$ & $\begin{array}{c}\mathrm{AV} \\
(\mathrm{dB} \mu V)\end{array}$ & $\begin{array}{c}\mathrm{QP} \\
(\mathrm{dB} \mu V)\end{array}$ & $\begin{array}{c}\mathrm{AV} \\
(\mathrm{dB} \mu V)\end{array}$ \\
\hline $\begin{array}{c}0.15-0.5 \\
\mathrm{MHz}\end{array}$ & 79 & 60 & $97-87$ & $84-74$ & $66-56$ & $56-46$ & $84-74$ & $74-64$ \\
\hline $0.5-5 \mathrm{MHz}$ & 73 & 66 & 87 & 74 & 56 & 46 & 74 & 64 \\
\hline $5-30 \mathrm{MHz}$ & & & & & 60 & 50 & & \\
\hline
\end{tabular}

Table 1: EN55022 conducted emission limits for class A and class B devices.

\section{$5 \quad$ EMC Analysis}

In this section, we present the setup and procedures of our experiments. In order to establish a PLC network, two computers were connected to two modems which 
are plugged into two sockets attached to the power line. Three measurement scenarios are involved, namely,

1. PLC modems are powered off to measure the noise.

2. PLC modems are on with no communication between the computers (i.e., Quiescent state),

3. PLC modems are switched on and data is active on the network (i.e., Busy state).

\subsection{Conducted Emission Measurements}

Common mode and differential mode measurements were taken using a current probe which is connected to the spectrum analyzer. Firstly, the common mode is measured in both states quiescent and busy (see Fig. 1a) and, similarly, the differential mode is measured in quiescent and busy states (see Fig. 1b). A simplified diagram of the conducted emission test setup is illustrated in Fig. 2.

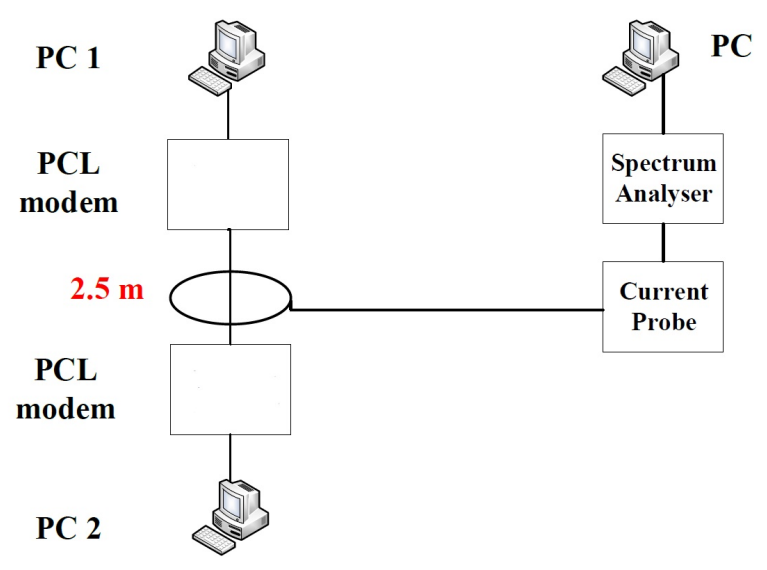

Fig. 2: Conducted emission test setup.

Radiated Emission Measurements Radiated measurements are carried out in this paper by a magnetic tracking antenna at a distance of $3 \mathrm{~m}$ from a $2.5 \mathrm{~m}$ power cable which connects the two computers as represented in Fig. 3. The data is collected and recorded on a PC which is already connected to the spectrum analyzer that is connected to the antenna. 


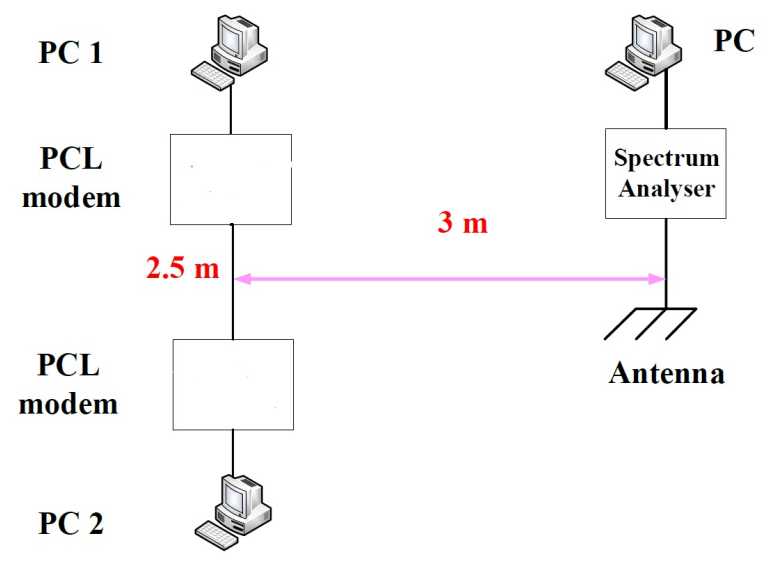

Fig. 3: Radiated emission test setup.

\section{$6 \quad$ Results and Discussions}

In this section we present and discuss the conducted and radiated emission results.

\subsection{Conducted Emissions}

To begin with, Fig. 4 presents the common and differential mode results over the frequency band $1 \mathrm{MHz}-30 \mathrm{MHz}$ for a TP-LINK modem. We can clearly see that the differential mode current is the dominant signal as it carries the desired date along the power cable. Although, the common mode current should not exist, we still can see its presence in Fig. 4. 


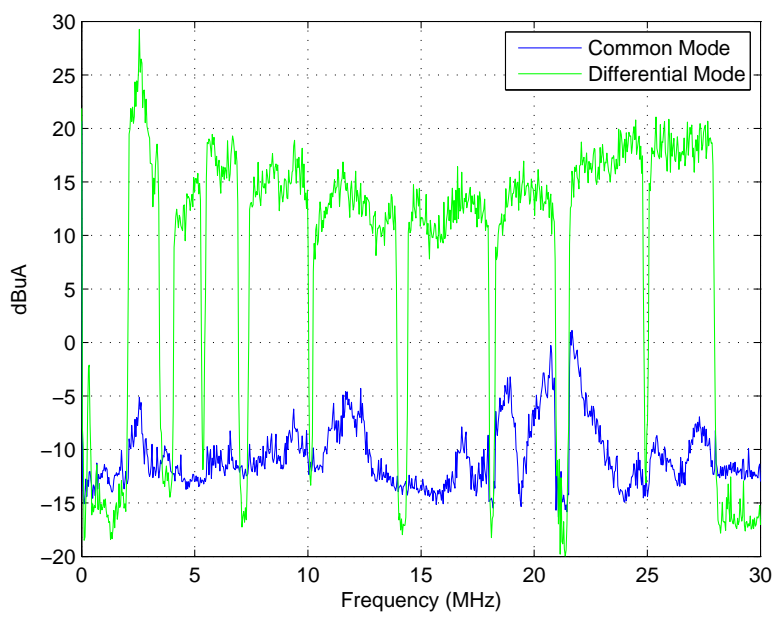

Fig. 4: Conducted current measurements for both the differential and common modes.

Fig. 5 illustrates the emission for busy state of the common and differential modes for both modems: TP-LINK and Homeplug Turbo. From Fig.5a and Fig.5b it is evident that in some cases, the readings of the TP-LINK are higher than the Homeplug Turbo measurements and vice versa. The conducted emission measurements in this figure are compared to the EN55022 main port limits and, as shown, there are three different values of the EN55022 limits depending on the operating frequency. However, the EN55022 limits are $32 \mathrm{~dB} \mu A$ for frequency range from $0.15 \mathrm{MHz}$ to $0.5 \mathrm{MHz}, 22 \mathrm{~dB} \mu \mathrm{A}$ for frequencies between $0.5 \mathrm{MHz}$ and $5 \mathrm{MHz}$ and for the frequencies from $5 \mathrm{MHz}$ to $30 \mathrm{MHz}$, it is $26 \mathrm{~dB} \mu A$. The common mode measurements indicate that the tested PLC modems do not exceed the EN55022 limits throughout the frequency spectrum under consideration. On the other hand, the differential mode measurements show that the TP-LINK exceeds the EN55022 limits between 2.5MHz and 3MHz. Furthermore, the EN55022 limits are exceeded by the differential mode readings of the Homeplug Turbo modem at frequency $22 \mathrm{MHz}$.

Moreover, the conducted emissions were measured in two different environments: a medium-sized Lab and a large lecture hall. In this respect, differential and common modes are compared in Fig. 6. From Fig. 6a, it is clear that, in most cases, the Lecture Hall measurements are dominant in the differential mode. Fig. $6 \mathrm{~b}$ shows the highest difference between both readings in common mode measurements is about $12 \mathrm{~dB} \mu \mathrm{A}$ where the Lab measurements are higher at some frequencies and the Lecture Hall measurements are higher at other frequencies. 


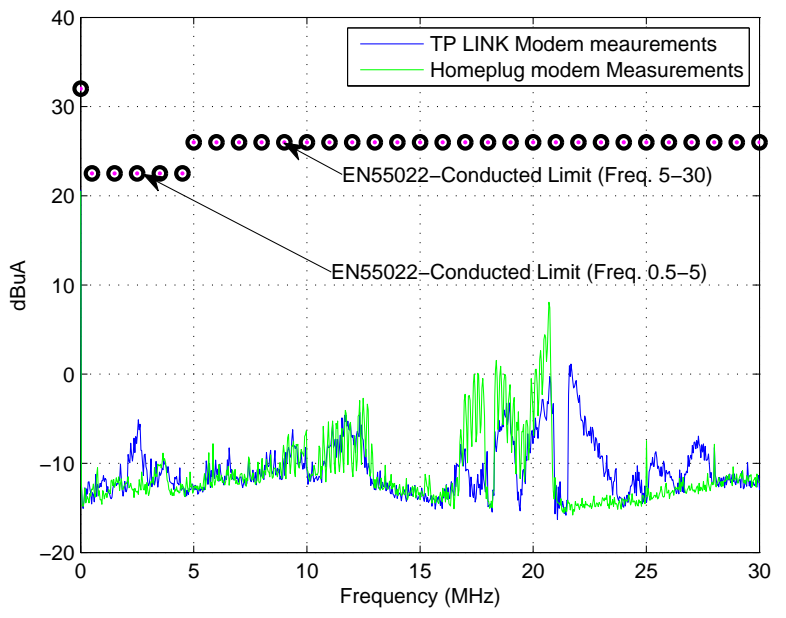

(a) Common mode.

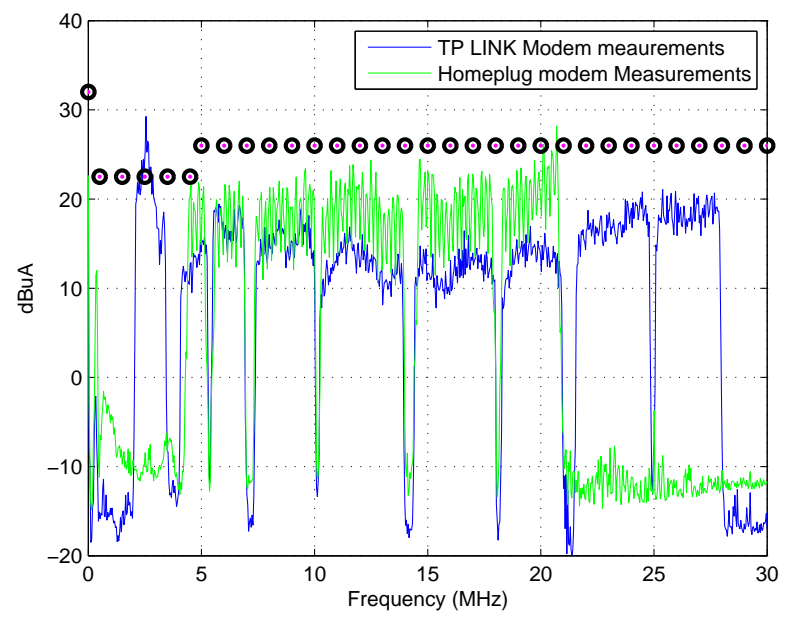

(b) Differential mode.

Fig. 5: Conducted emissions during busy state of the common and differential modes for TP-LINK and Homeplug Turbo modems compared to the EN55022 limits. 


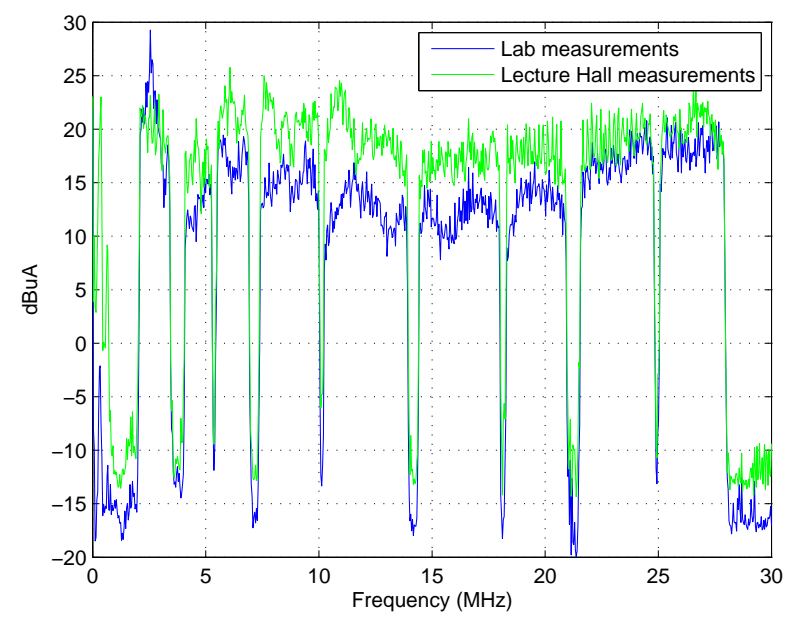

(a) Differential mode

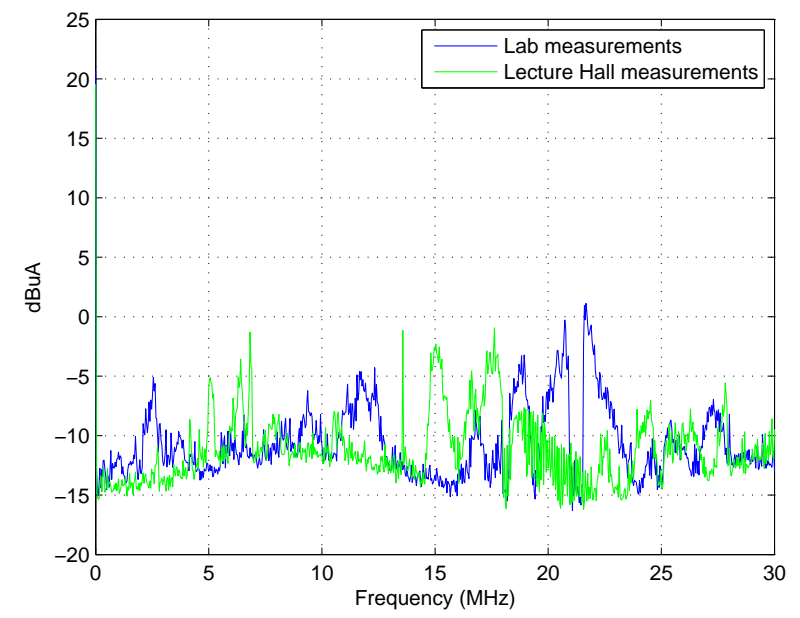

(b) Common mode

Fig. 6: Conducted emission measurements for the TP-LINK modem in two different indoor environments. 


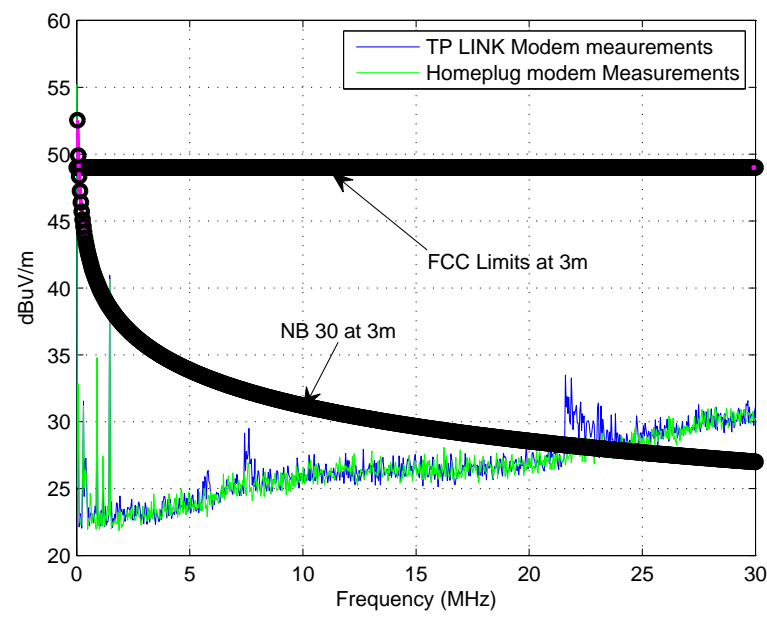

Fig. 7: Radiated emission measurements and their comparison to the standards.

\subsection{Radiated Emission}

Fig. 7 depicts the radiated emission for both modems under consideration. Clearly, the peak of the field strength is about $45 \mathrm{~dB} \mu V / m$ which occurs at very low frequency. These results are compared to the NB30 and FCC standards. It is noticeable that the measurements of both modems do not exceed the FCC limits in the frequency band $0-30 \mathrm{MHz}$. Both measurements indicate that the used PLC systems do not comply with NB30 limits as the TP-LINK modem exceeds these limits at frequencies around $2 \mathrm{MHz}$ and above $23 \mathrm{MHz}$. Moreover, all results taken at almost $22 \mathrm{MHz}$ and above exceed the NB30 limits for Homeplug Turbo modem. It should be pointed out that these measurements are compared to the standards without considering the antenna calibration factor which should be added to the measurements before comparing them to the standards. This factor is called antenna factor; it is treated as a loss in $\mathrm{dB}$. Also, a cable losses ought to be considered and added to the readings.

The final set of results is shown in Fig. 8. This figure shows a comparison between the radiated emissions of TP-LINK modem in two different environments, i.e., lab and lecture hall. It is clear that the lecture hall measurements are higher than the lab readings for the frequency range $0-7 \mathrm{MHz}$ whereas the emissions in the lab are dominant in the frequency range $22-24 \mathrm{MHz}$.

\section{Conclusion}

From an overall perspective to the measurements taken in this frame work, it was confirmed that the tested PLC modems totally comply with FCC limits for the radiated emmisions and with the EN55022 in most cases for the conducted 
one. In fact, there was at least a minimum of $10 \mathrm{~dB}$ margin between our radiated measurements and the FCC regulation standards; this margin reaches as high as $35 \mathrm{~dB}$ at some frequencies. On the other hand, the comparison between measured radiated emissions and the standards showed that the former exceeds one of the proposed regulation limits. In some measured radiated emissions, the field strength curve exceeds the NB30 limits. Comparisons of both conducted and radiated emissions in different environments were presented. It was clear that emissions were affected by the place and surrounding areas where the measurements took place.

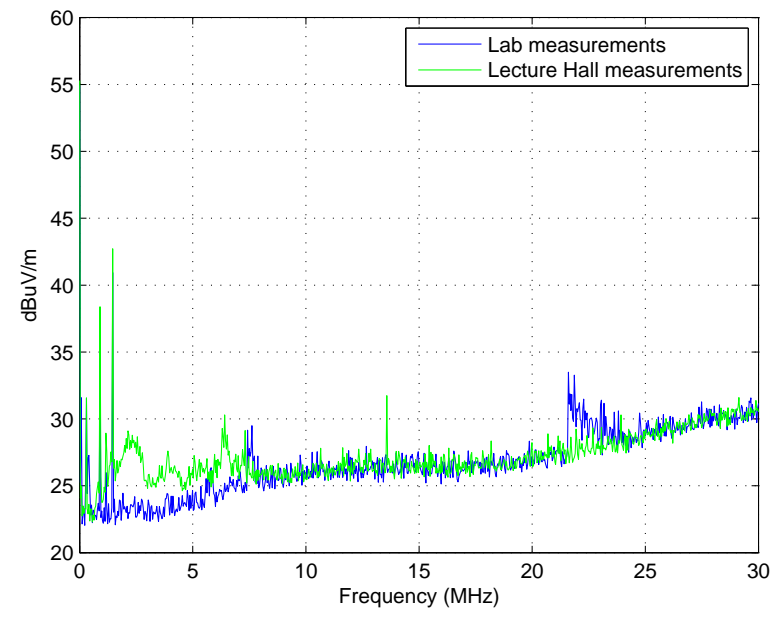

Fig. 8: Radiated emissions in two different indoor environments.

\section{Acknowledgment}

This research has been carried out within the "CityVerve: IoTs and Smart Cities Demonstrator" project funded by Innovate UK (102561).

\section{References}

1. B. Adebisi and B. Honary. Comparisons of indoor PLC emissions measurement results and regulation standards. In Proc. IEEE Int. Symp. Power Line Commun., pages 319-324, 2006.

2. B. Adebisi, K. M. Rabie, A. Ikpehai, C. Soltanpur, and A. Wells. Vector ofdm transmission over non-gaussian power line communication channels. IEEE Systems Journal, PP(99):1-9, 2017. 
3. B. Adebisi, J. Stott, and B. Honary. Experimental study of the interference caused by PLC transmission on HF bands. In IET Int. Conf. Ionospheric Radio Systems and Techniques (IRST), pages 326-330, Jul. 2006.

4. M. Girotto and A. M. Tonello. Emc regulations and spectral constraints for multicarrier modulation in plc. IEEE Access, 5:4954-4966, 2017.

5. F. Grassi, S. A. Pignari, and J. Wolf. Channel characterization and emc assessment of a plc system for spacecraft dc differential power buses. IEEE Trans. Electromagn. Compat., 53(3):664-675, Aug. 2011.

6. D. Hansen. Review of emc aspects in recent european plc development. In IEEE Power Engineering Society Summer Meeting,, volume 3, pages 1200-1216 vol.3, Jul. 2002.

7. H. Meng, Y. L. Guan, and S. Chen. Modeling and analysis of noise effects on broadband power-line communications. IEEE Trans. Power Del., 20(2):630-637, Apr. 2005.

8. D. Middleton. Statistical-physical models of electromagnetic interference. IEEE Trans. Electromagn. Compat., EMC-19(3):106-127, Aug. 1977.

9. M. Rozman, A. Ikpehai, B. Adebisi, and K. M. Rabie. Channel characterisation of cooperative relaying power line communication systems. In 10th International Symposium on Communication Systems, Networks and Digital Signal Processing (CSNDSP), pages 1-5, Jul. 2016.

10. M. Tlich, R. Razafferson, G. Avril, and A. Zeddam. Outline about the emc properties and throughputs of the plc systems up to $100 \mathrm{mhz}$. In IEEE Int. Symp. PLC Techniques $\&$ Applications, pages 259-262, April 2008.

11. H. Widmer. On the global emc aspect of broadband power line communications using the ldquo;hf rdquo; frequency band. In 2000 International Zurich Seminar on Broadband Communications. Accessing, Transmission, Networking. Proceedings (Cat. No.00TH8475), pages 179-184, 2000. 